Copyright (C) 2009 IEEE. Reprinted from $31^{\text {st }}$ Annual International Conference of the IEEE Engineering in Medicine and Biology Society, 2009. EMBC 2009. Sept. 2009.

This material is posted here with permission of the IEEE. Such permission of the IEEE does not in any way imply IEEE endorsement of any of Cornell University's products or services. Internal or personal use of this material is permitted. However, permission to reprint/republish this material for advertising or promotional purposes or for creating new collective works for resale or redistribution must be obtained from the IEEE by writing to pubs-permissions@ieee.org.

By choosing to view this document, you agree to all provisions of the copyright laws protecting it. 


\title{
Estimation of Anatomical Locations using Standard Frame of Reference in Chest CT Scans
}

\author{
Jaesung Lee, Alberto M. Biancardi, Anthony P. Reeves, David F. Yankelevitz, and Claudia I. Henschke
}

\begin{abstract}
We propose a method to establish the standard chest frame of reference (CFOR) using the rib cage in a lung CT scan. Such a reference frame is essential for referring to a certain location within a chest region and may facilitate the registration across multiple scans of a given subject as well as the comparative studies within a cohort of subjects. The robustness of the established CFOR was evaluated by estimating the anatomical locations within chest in the follow-up scan given the location in the first scan. Specifically, tracheal bifurcation point of airway tree and the center of pulmonary nodule were used as the anatomical points of interest. The CFOR was also used for exploring the spatial distribution of the anatomical location for a large number of individuals. The results show that on average the point of interest can be estimated accurately within $10.3 \mathrm{~mm}$ for the bifurcation point and within $12.5 \mathrm{~mm}$ for the pulmonary nodule's center point. Further analyzing the spatial distribution of the CFOR coordinates across 86 subjects shows that we can localize the bifurcation point to the small subregion within the CFOR.
\end{abstract}

Index Terms-anatomical location, frame of reference, computed tomography (CT), chest imaging

\section{INTRODUCTION}

In order to refer to a certain location or perform anatomical matching for different CT scans, it is necessary to establish the frame of reference that is standardized for any CT scans. Such a reference frame may facilitate the registration across multiple scans of a given subject as well as the comparative studies within a cohort of subjects.

Among many anatomical structures within a human body, the bone structure may serve as a solid reference point for locating other anatomical parts in a given CT scan. In this paper a computer algorithm is presented which, given a CT scan, automatically establishes a chest frame of reference (CFOR) based on the rib cage. Robustness of the established frame is explored using the locations of tracheal bifurcation points and pulmonary nodules.

The technique that is closely related to this paper is the segmentation-based registration, where anatomically the same structures are extracted from both images to be registered and used as sole input for the alignment procedure [1]. While there has not been much work for a standardized frame of reference for the entire chest region, there were

This work was supported in part by NIH grant R33CA101110 and a grant from Carestream Health, Inc.

J. Lee, A. Biancardi, and A. Reeves are with the School of Electrical and Computer Engineering, Cornell University, Ithaca, NY, USA. j1569@cornell.edu

D. Yankelevitz and C. Henschke are with the Department of Radiology, Weill Cornell Medical College, New York, NY, USA. considerable interest in the registration of a local region of interest such as pulmonary nodule across multiple image data. [2][3][4]

In related work Shi et al. [2] developed an automated registration method for identifying corresponding pulmonary nodules in serial CT scans. Their method performed the initial nodule registration using the ribs as reference and further refined it by searching for the best match between the volumes of interest. Betke et al. [3] performed the registration of lung surfaces using the detected landmarks. They then applied the lung registration to locate corresponding nodules in the serial CT scans. Kawata et al. [4] developed a two-step method for matching nodules using rigid-body registration and affine transformation.

Previous works have been focused on the registration of a local structure, such as a pulmonary nodule, across a scan pair. The work presented in this paper focuses on establishing a standard frame of reference for the entire chest region which may be used for both intra- and inter- subject evaluations.

\section{Chest Frame of Reference (CFOR)}

The chest frame of reference is defined with the coordinate system where for each dimension the frame extent ranges from -1.0 to +1.0 . The reference frame is built based on the rib bones, and some anatomical structures may be located outside of the frame. The frame is defined for each dimension as following:

- Axial direction of the chest $(\mathrm{z})$ : the bottom-most rib root will be -1.0 and the top-most rib root will be +1.0.

- Sagittal direction of the chest $(\mathrm{x})$ : the patient's rightmost extent of the rib cage will be -1.0 and the left-most extent of the rib cage will be +1.0 .

- Coronal direction of the chest (y): the most anterior extent of the rib cage will be -1.0 and the most posterior extent of the rib cage will be +1.0 .

Figure 1 shows how a frame of reference is defined for a chest CT scan. For each scan we define a scale-offset locator (SOL), composed of a scale matrix $\mathbf{S}$ and an offset vector $\mathbf{o}$ as defined in the equations 1 and 2 :

$$
\begin{gathered}
\mathbf{S}=\operatorname{diag}\left(\begin{array}{lll}
x s & y s & z s
\end{array}\right), \\
\mathbf{o}=\left(\begin{array}{lll}
\operatorname{xoff} & y o f f & z o f f
\end{array}\right)^{T} .
\end{gathered}
$$

An SOL is defined in terms of millimeters and can be used to map a point between the scan's original coordinate system and the chest frame of refrence. An image coordinate may 


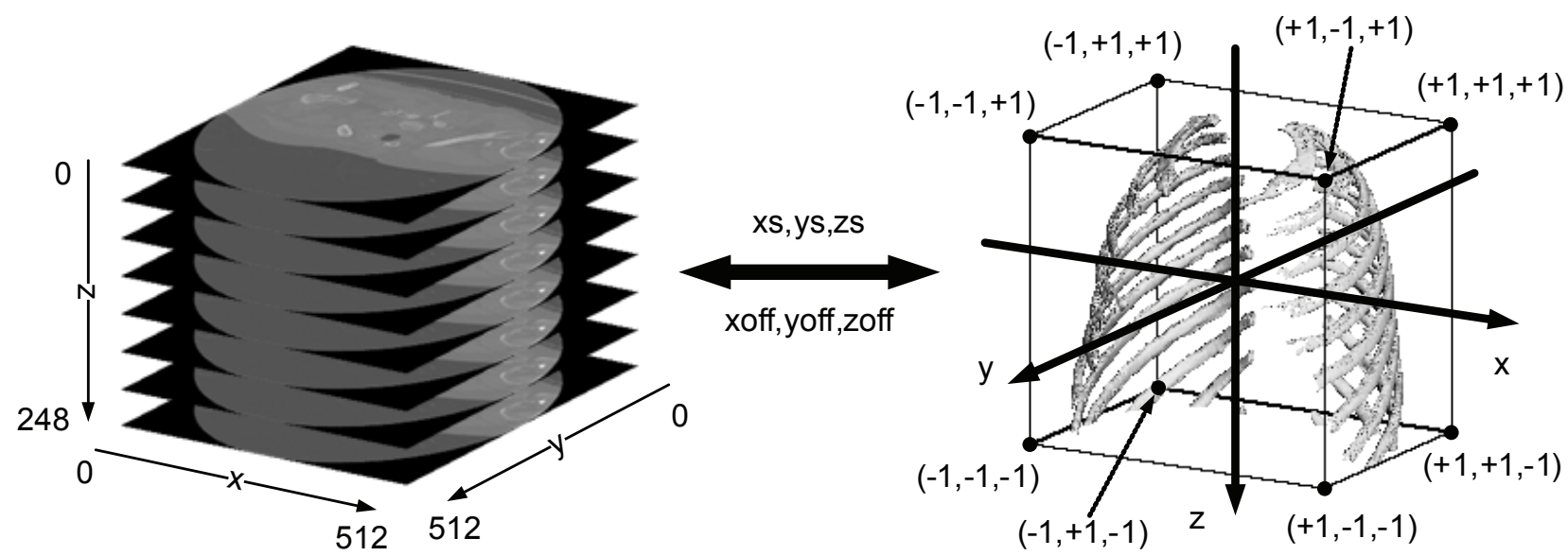

Fig. 1. Chest frame of reference. A point in a 3D volumetric image (left) can be mapped to a point in the standardized frame of reference (right) using the SOL (scale-offset matrices). An SOL is defined for each CT scan using the segmented rib cage. Note that coordinates outside of \pm 1 extent for the frame of reference are valid as some parts of the CT image may lie outside of the frame.

be mapped to a CFOR coordinate using Equation 3, and vice versa using Equation 4:

$$
\begin{gathered}
\mathbf{c}_{c f o r}=\mathbf{S} *\left(\mathbf{c}_{i m g}+\mathbf{o}\right), \\
\mathbf{c}_{i m g}=\left(\mathbf{S}^{-1} * \mathbf{c}_{c f o r}\right)-\mathbf{o},
\end{gathered}
$$

where $\mathbf{c}_{i m g}$ is an image coordinate in millimeters, and $\mathbf{c}_{c f o r}$ is a CFOR coordinate.

\section{Method AND MATERiALs}

\section{A. Establishing CFOR}

The chest frame of reference is established based on the rib bone locations for a given image. The entire bone structure present in a CT scan is obtained by first filtering the scan with a $3 \times 3$ mean filter and thresholding. The threshold level was chosen to be low enough to include all bone structures, and small high-intensity voxels were removed after thresholding.

To segment the ribs the centerline of the spinal canal is traced using a distance transform on the bone images. The rib starting points are then identified based on the spinal canal location. Starting from each rib root, 3D region growing is used to segment the individual rib bone.

Once the rib bones are segmented for the given scan, a chest frame of reference is established by computing an SOL as described in Section II.

\section{B. Experimental Design}

The experiments were set up for both intra-subject and inter-subject evaluations. The intra-subject evaluation involved the serial CT scan pairs of the same subject, and the inter-subject evaluation involved the CT scans for a cohort of patients.

1) Intra-subject Evaluation: In this part, the experiment was set up to evaluate the robustness of CFOR scheme for identifying the corresponding anatomical locations in a pair of serial CT scans. The overview of the intra-subject experiment is outlined as a flowchart in Figure 2.

The anatomical point in the first scan was mapped to a point in CFOR, and the location of corresponding point

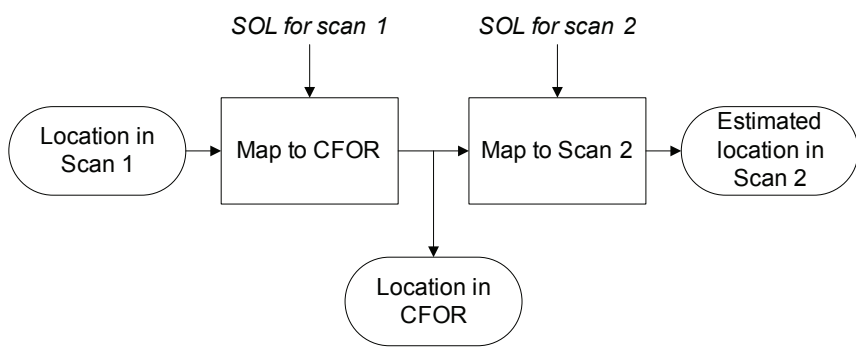

Fig. 2. Overview of intra-subject evaluation. Given a location in the first scan, the corresponding location in the second scan is estimated using the chest frame of reference.

was estimated in the second scan by mapping the CFOR coordinate to the second scan. The accuracy of the estimated location was quantified with the distance to the actual location in the second scan.

Since the CFOR was defined separately for each scan, different prediction error was obtained when two scans were swapped (i.e. the second scan's location is mapped to the first one). Since the purpose was to establish a standardized coordinate system for any scan regardless of the acquisition time, the errors for the worst case were reported in the paper.

The CFOR was evalulated using two anatomical locations: tracheal bifurcation point and center of pulmonary nodule.

- Bifurcation Point of Trachea: The bifurcation point was defined in this paper as the point at which the centerline of the airway tree diverges. For each scan the tracheal bifurcation point was automatically detected using a method described in [5].

- Center of Pulmonary Nodule: The pulmonary nodules with the diameters above $2.5 \mathrm{~mm}$ were considered for the experiment. The center of the nodule was manually identified in each scan.

2) Inter-subject Evaluation: In this part of the experiment, inter-subject variability of an anatomical location was explored. Specifically, the bifurcation point of trachea was localized to a subregion within the CFOR. The inter-subject 


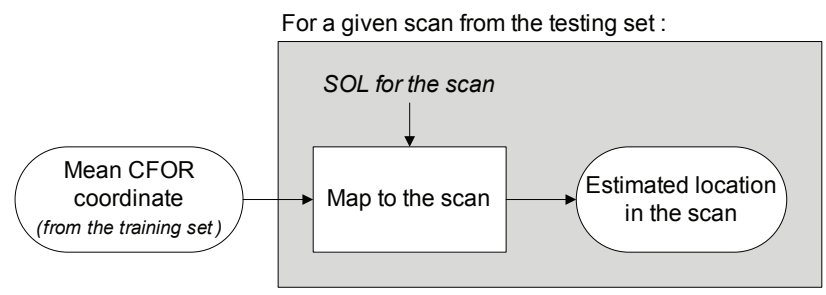

Fig. 3. Overview of inter-subject evaluation. Given a mean CFOR coordinate obtained from the training data, the corresponding location is estimated for the scans in the testing set.

experiment overview is shown as a flowchart in Figure 3.

First the dataset was divided into the training and testing sets, and the mean CFOR coordinate of trachea bifurcation point was obtained from a training dataset of CT scans. Then the mean CFOR coordinate was mapped to the testing set, and the distance to the actual location was computed.

\section{Dataset}

All CT scans used for the experiments were whole-lung CT scans taken with low radiation dose $(120 \mathrm{kV}, 40 \mathrm{mAs})$ and had the slice thickness of $1.25 \mathrm{~mm}$. In-plane resolution of the scans ranged from $0.55 \times 0.55 \mathrm{~mm}$ to $0.82 \times 0.82 \mathrm{~mm}$. The time interval and lung volume change between the scan pair for the intra-subject experiment is shown in Table I. The nodule location was expected to be sensitive to the inspiration, and the cases with the lung volume change greater than $20 \%$ were excluded from the analysis.

TABLE I

DATASET FOR INTRA-SUBJECT EVALUATION

\begin{tabular}{|c||c|c|}
\hline & Bifurcation point & Nodule center \\
\hline \hline Time interval (days) & $411 \pm 344(\mathrm{SD})$ & $667 \pm 473(\mathrm{SD})$ \\
\hline Lung volume difference $(\%)$ & $4.8 \pm 5.2(\mathrm{SD})$ & $6.6 \pm 6.7(\mathrm{SD})$ \\
\hline
\end{tabular}

136 scan pairs were used for the intra-subject evaluation of the bifurcation point. For the nodule's center point, 21 scan pairs were used, where a benign pulmonary nodule was manually located in each scan pair. The first scans of 136 scan pairs were also used for inter-subject evaluation of the bifurcation point, of which 50 were used as the training data.

\section{Results}

Table II shows the deviation of the estimated point from the actual location. The Euclidean distance is reported along with the deviations in $\mathrm{x}, \mathrm{y}$, and $\mathrm{z}$ dimensions. On average the bifurcation point was located within $10.3 \mathrm{~mm}$ from the estimated point, and the nodule center was correctly estimated within $12.5 \mathrm{~mm}$. For both anatomical points the prediction errors were plotted against the time intervals in Figures 4 and 5.

The average CFOR coordinate of the tracheal bifurcation point, computed from the training data, was $(-0.043,-0.007$, -0.416). Table III shows the deviation of the actual point from the average location for 86 subjects. On average the

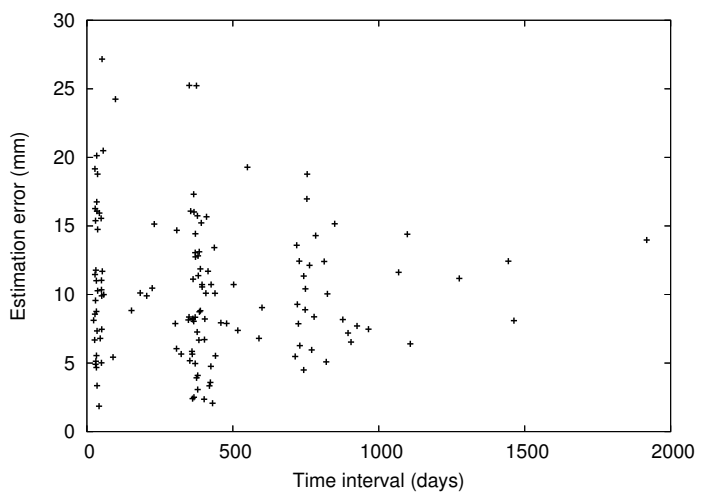

Fig. 4. Prediction error for the tracheal bifurcation point. $(n=136)$ The Euclidean distance is plotted against the time interval between two scans.

bifurcation point was located within $17.3 \mathrm{~mm}$ from the mean location obtained from the training set. The distribution of the distances from the average location is shown as a histogram in Figure 6.

TABLE II

INTRA-SUBJECT PREDICTION ERRORS FOR ANATOMICAL LOCATIONS $(\mathrm{MEAN} \pm \mathrm{SD})$

\begin{tabular}{|c||c|c|}
\hline Measure & Bifurcation point $(\mathrm{mm})$ & Nodule center $(\mathrm{mm})$ \\
\hline \hline Euclidean distance & $10.28 \pm 5.02$ & $12.45 \pm 5.81$ \\
\hline Distance in $\mathrm{X}$ & $5.80 \pm 4.66$ & $5.78 \pm 4.40$ \\
\hline Distance in $\mathrm{Y}$ & $4.96 \pm 3.71$ & $5.54 \pm 4.92$ \\
\hline Distance in Z & $4.49 \pm 4.16$ & $7.48 \pm 5.17$ \\
\hline
\end{tabular}

TABLE III

INTER-SUBJECT ESTIMATION OF BIFURCATION POINT $(\mathrm{MEAN} \pm \mathrm{SD})$

\begin{tabular}{|c||c|}
\hline Measure & Inter-subject variation (mm) \\
\hline \hline Euclidean distance & $17.25 \pm 7.45$ \\
\hline Distance in $\mathrm{X}$ & $5.88 \pm 4.80$ \\
\hline Distance in $\mathrm{Y}$ & $8.64 \pm 5.88$ \\
\hline Distance in $\mathrm{Z}$ & $11.02 \pm 8.11$ \\
\hline
\end{tabular}

\section{DISCUSSION}

The CFOR scheme was evaluated for its robustness across the scan pair in the intra-subject experiment. Two anatomical points of interest used in the paper included the bifurcation point of a trachea and the center of a nodule. Both anatomical points were estimated with the errors less than $1.5 \mathrm{~cm}$. The bifurcation point was estimated with slightly less error $($ mean $=1.0 \mathrm{~cm})$ than the nodule center $($ mean $=1.2 \mathrm{~cm})$.

The prediction errors were plotted against the time interval between the scans for both anatomical points (Figures 4 and 


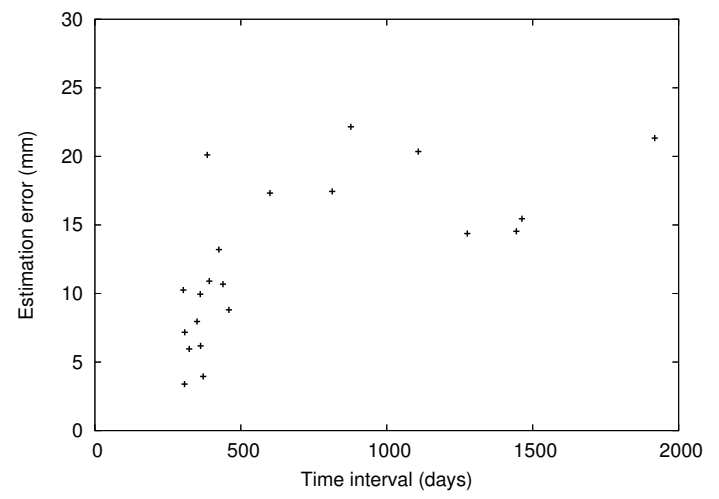

Fig. 5. Prediction error for the nodule center. $(n=21)$ The Euclidean distance is plotted against the time interval between two scans.

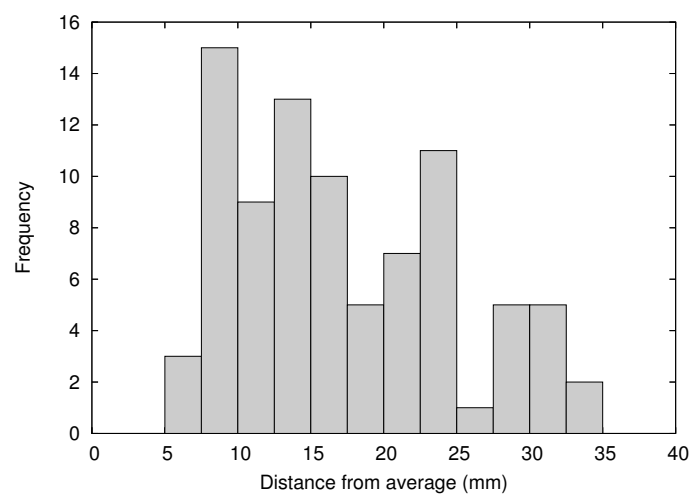

Fig. 6. Histogram of the distances from the average bifurcation point location for the inter-subject evaluation. $(n=86)$

5). There were low correlations between the time interval and the prediction error for both anatomical points. Further analysis indicated that neither of the lung volume change across the scans and the vertical position of the point (i.e. $\mathrm{z}$ coordinate) had a significant effect on the prediction errors.

The prediction error of $1.3 \mathrm{~cm}$ for the nodule location may be relatively large considering that the mean diameter of the nodules used in the experiment was around $7 \mathrm{~mm}$. However, lung is a non-rigid structure and its morphology may change while breathing. Starting from the estimated point the nodules may be further registered using a template matching as shown in [2].

The focus of this work was to establish a standardized chest frame of reference for identifying an anatomical region of interest within entire chest area and provide a framework that may facilitate further registration. The proposed CFOR scheme is preliminary as it does not account for possible rotation of a patient body. The orientation of the body may affect how a CFOR is defined and account for some prediction errors. The preliminary results show that the proposed CFOR scheme works well in providing with the standardized coordinate system.

Figure 7 shows the relationship between the prediction errors for the bifurcation point and the nodule center. The prediction errors were plotted for 21 cases in which the

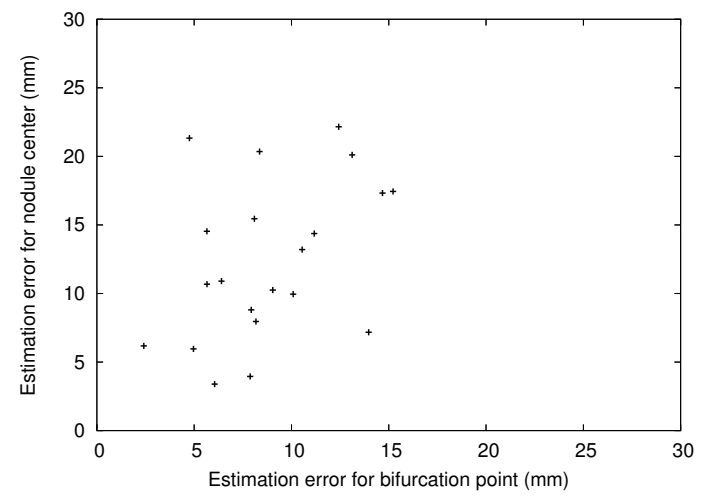

Fig. 7. Relationship between the prediction errors for the bifurcation point and the nodule center. $(n=21)$

nodules were identified. The plot shows that the bifurcation points are better estimated than the nodules but indicates low correlation between the two prediction errors, suggesting that the prediction errors for the two points are not caused by a common scan attribute.

In the second experiment the inter-subject variability of the bifurcation point location was evaluated using the CFOR. On average the actual bifurcation point deviated by $1.7 \mathrm{~cm}$ from the pre-established estimation based on the training data. The most variation was observed in $\mathrm{z}$ direction of the scan (mean=11.0 mm), and the least variation was in $\mathrm{x}$ direction (mean=5.9 mm).

\section{CONCLUSIONS}

When referring to a certain location in different chest CT scans, it is necessary to have a standardized coordinate system. In this paper, a method to establish the standard chest frame of reference (CFOR) was presented, which builds the CFOR based on the segmented rib cage for a given CT scan. The CFOR scheme was used for both intra- and inter-subject experiments for locating the anatomical points of interest. The results show that the CFOR scheme is able to localize the point of interest to the small subregion across different scans and therefore serves as a well-established standard coordinate system.

\section{REFERENCES}

[1] J. Maintz and M. Viergever, "A survey of medical image registration," Medical Image Analysis, vol. 2, no. 1, pp. 1-36, 1998.

[2] J. Shi, B. Sahiner, H. Chan, L. Hadjiiski, C. Zhou, P. Cascade, N. Bogot, E. Kazerooni, Y. Wu, and J. Wei, "Pulmonary nodule registration in serial CT scans based on rib anatomy and nodule template matching," Medical Physics, vol. 34, p. 1336, 2007.

[3] M. Betke, H. Hong, D. Thomas, C. Prince, and J. Ko, "Landmark detection in the chest and registration of lung surfaces with an application to nodule registration," Medical Image Analysis, vol. 7, no. 3, pp. 265281, 2003.

[4] Y. Kawata, N. Niki, H. Omatsu, M. Kusumoto, R. Kakinuma, K. Mori, H. Nishiyama, K. Eguchi, M. Kaneko, and N. Moriyama, "Tracking interval changes of pulmonary nodules using a sequence of threedimensional thoracic images," in Proceedings of SPIE Medical Imaging, vol. 3979,2000 , p. 86.

[5] J. Lee, A. Reeves, D. Yankelevitz, and C. Henschke, "Bronchial segment matching in low-dose lung CT scan pairs," in Proceedings of SPIE Medical Imaging, vol. 7260, 2009, p. 72600A. 